\title{
Interactive Quadratic Gravity
}

\author{
K Kleidis, A Kuiroukidis and D B Papadopoulos \\ Department of Physics \\ Section of Astrophysics, Astronomy and Mechanics \\ Aristotle University of Thessaloniki \\ 54006 Thessaloniki, Greece
}

\begin{abstract}
A quadratic semiclassical theory, regarding the interaction of gravity with a massive scalar quantum field, is considered in view of the renormalizable energymomentum tensor in a multi-dimensional curved spacetime. According to it, a self-consistent coupling between the square curvature term $\mathcal{R}^{2}$ and the quantum field $\Phi(t, \vec{x})$ should be introduced in order to yield the "correct" renormalizable energy-momentum tensor in quadratic gravity theories. The subsequent interaction discards any higher-order derivative terms from the gravitational field equations, but, in the expence, it introduces a geometric source term in the wave equation for the quantum field. Unlike the conformal coupling case $\left(\sim \mathcal{R} \Phi^{2}\right)$, this term does not represent an additional "mass" and, therefore, the quantum field interacts with gravity not only through its mass (or energy) content $\left(\sim \Phi^{2}\right)$, but, also, in a more generic way $\left(\mathcal{R}^{2} \Phi\right)$. Within this context, we propose a general method to obtain mode-solutions for the quantum field, by means of the associated Green's function in an anisotropic six-dimensional background.
\end{abstract}

\section{Introduction}

In the last few decades there has been a remarkable progress in understanding the quantum structure of the non-gravitational fundamental interactions (Nanopoulos 1997). On the other hand, so far, there is no quantum framework consistent enough to describe gravity itself (Padmanabhan 1989), leaving string theory as the most successful attempt towards this direction (Green et al 1987, Polchinsky 1998, Schwarz 1999). Within the context of General Relativity (GR), one usually resorts to the perturbative approach, where string theory predicts corrections to the Einstein equations. Those corrections originate from higher-order curvature terms arising in the string action, but their exact form is not yet being fully explored (Polchinsky 1998). 
A self-consistent mathematical background for higher-order gravity theories was formulated by Lovelock (Lovelock 1971). According to it, the most general gravitational Lagrangian reads

$$
\mathcal{L}=\sqrt{-g} \sum_{m=0}^{n / 2} \lambda_{m} \mathcal{L}^{(m)}
$$

where $\lambda_{m}$ are constant coefficients, $n$ denotes the spacetime dimensions, $g$ is the determinant of the metric tensor and $\mathcal{L}^{(m)}$ are functions of the Riemann curvature tensor $\mathcal{R}_{i j k l}$ and its contractions $\mathcal{R}_{i j}$ and $\mathcal{R}$, of the form

$$
\mathcal{L}^{(m)}=\frac{1}{2^{m}} \delta_{i_{1} \ldots i_{2 m}}^{j_{1} \ldots j_{2 m}} \mathcal{R}_{j_{1} j_{2}}^{i_{1} i_{2}} \ldots \mathcal{R}_{j_{2 m-1} j_{2 m}}^{i_{2 m-1} i_{2 m}}
$$

where Latin indices refer to the n-dimensional spacetime and $\delta_{i_{1} \ldots i_{2 m}}^{j_{1} \ldots j_{2 m}}$ is the generalized Kronecker symbol. In Eq. (2), $\mathcal{L}^{(1)}=\frac{1}{2} \mathcal{R}$ is the Einstein-Hilbert (EH) Lagrangian, while $\mathcal{L}^{(2)}$ is a particular combination of quadratic terms, known as the Gauss-Bonnett (GB) combination, since in four dimensions it satisfies the functional relation

$$
\frac{\delta}{\delta g^{\mu \nu}} \int \sqrt{-g}\left(\mathcal{R}^{2}-4 \mathcal{R}_{\mu \nu} \mathcal{R}^{\mu \nu}+\mathcal{R}_{\mu \nu \kappa \lambda} \mathcal{R}^{\mu \nu \kappa \lambda}\right) d^{4} x=0
$$

corresponding to the GB theorem (Kobayashi and Nomizu 1969). In Eq. (3), Greek indices refer to four-dimensional coordinates. Introducing the GB term into the gravitational Lagrangian will not affect the four-dimensional field equations at all. However, within the context of the perturbative approach mentioned above, the most important contribution comes from the GB term (Mignemi and Stewart 1993). As in four dimensions it is a total divergence, to render this term dynamical, one has to consider a higher-dimesional background or to couple it to a scalar field.

The idea of a multi-dimensional spacetime has received much attention as a candidate for the unification of all fundamental interactions, including gravity, in the framework of supergravity and superstrings (Applequist et al 1987, Green et al 1987). In most higher-dimensional theories of gravity, the extra dimensions are assumed to form, at the present epoch, a compact manifold (internal space) of very small size compared to that of the three-dimensional visible space (external space) and therefore they are unobservable at the energies currently available (Green et al 1987). This so-called compactification of the extra dimensions may be achieved, in a natural way, by adding a square-curvature term $\left(\mathcal{R}_{i j k l} \mathcal{R}^{i j k l}\right)$ in the EH action of the gravitational field (Müller-Hoissen 1988). In this way, the higher-dimensional theories are closely related to those of non-linear Lagrangians and their combination probably yields a natural generalization of GR.

In the present paper, we explore this generalization in view of the renormalizable energy-momentum tensor which acts as the source of gravity in the (semiclassical) interaction of the gravitational with a quantum matter field. In particular: 
In Section II, we discuss briefly how GR is modified by the introduction of the renormalizable energy-momentum tensor first recognized by Calan et al (1970), on the rhs of the field equations. Introducing an analogous method, we explore the corresponding implications as regards a multi-dimensional higher-order gravity theory. We find that, in this case, the action functional, describing the semi-classical interaction of a quantum scalar field with the classical gravitational one, is being further modified and its variation with respect to the quantum field results in an inhomogeneous KleinGordon equation, the source term of which is purely geometric $\left(\sim \mathcal{R}^{2}\right)$. Accordingly, using a general method, we solve this equation, by means of the associated Green's function, in a compact, anisotropic six-dimensional background (Section III).

\section{Quadratic Interaction}

Conventional gravity in $n$-dimensions implies that the dynamical behaviour of the gravitational field arises from an action principle involving the EH Lagrangian

$$
\mathcal{L}_{E H}=\frac{1}{16 \pi G_{n}} \mathcal{R}
$$

where $G_{n}=G V_{n-4}$ and $V_{n-4}$ denotes the volume of the internal space, formed by some extra spacelike dimensions. In this framework, we consider the semi-classical interaction between the gravitational and a massive quantum scalar field $\Phi(t, \vec{x})$ to the lowest order in $G_{n}$. The quantization of the field $\Phi(t, \vec{x})$ is performed by imposing canonical commutation relations on a hypersurface $t=$ constant (Isham 1981)

$$
\begin{gathered}
{\left[\Phi(t, \vec{x}), \Phi\left(t, \vec{x}^{\prime}\right)\right]=0=\left[\pi(t, \vec{x}), \pi\left(t, \vec{x}^{\prime}\right)\right]} \\
{\left[\Phi(t, \vec{x}), \pi\left(t, \vec{x}^{\prime}\right)\right]=i \delta^{(n-1)}\left(\vec{x}-\vec{x}^{\prime}\right)}
\end{gathered}
$$

where $\pi(t, \vec{x})$ is the momentum canonically conjugate to the field $\Phi(t, \vec{x})$. The equaltime commutation relations (5) guarantee the local character of the quantum field theory under consideration, thus attributing its time-evolution to the classical gravitational field equations (Birrell and Davies 1982).

In any local field theory, the corresponding energy-momentum tensor is a very important object. Knowledge of its matrix elements is necessary to describe scattering in a relatively-weak external gravitational field. Therefore, in any quantum process in curved spacetime, it is desirable for the corresponding energy-momentum tensor to be renormalizable; i.e. its matrix elements to be cut-off independent (Birrell and Davies 1982). In this context, it has been proved (Callan et al 1970) that the functional form of the renormalizable energy-momentum tensor involved in the semi-classical interaction between the gravitational and a quantum field in $n$-dimensions, should be

$$
\Theta_{i k}=T_{i k}-\frac{1}{4} \frac{n-2}{n-1}\left[\Phi_{; i k}^{2}-g_{i k} \square \Phi^{2}\right]
$$


where the semicolon stands for covariant differentiation $\left(\nabla_{k}\right), \square=g^{i k} \nabla_{i} \nabla_{k}$ is the d' Alembert operator and

$$
T_{i k}=\Phi_{, i} \Phi_{, k}-g_{i k} \mathcal{L}_{m a t}
$$

is the conventional energy-momentum tensor of an (otherwise) free massive scalar field, with Lagrangian density of the form

$$
\mathcal{L}_{\text {mat }}=\frac{1}{2}\left[g^{i k} \Phi_{, i} \Phi_{, k}-m^{2} \Phi^{2}\right]
$$

It's worthnoting that the tensor (6) defines the same $n$-momentum and Lorentz generators as the conventional energy-momentum tensor.

It has been shown (Callan et al 1970) that the energy-momentum tensor (6) can be obtained by an action principle, involving

$$
S=\int\left[f(\Phi) \mathcal{R}+\mathcal{L}_{\text {mat }}\right] \sqrt{-g} d^{n} x
$$

where $f(\Phi)$ is an arbitrary, analytic function of $\Phi(t, \vec{x})$, the determination of which can be achieved by demanding that the rhs of the field equations resulting from Eq. (9) is given by Eq. (6). Accordingly,

$$
\frac{\delta S}{\delta g^{i k}}=0 \Rightarrow \mathcal{R}_{i k}-\frac{1}{2} g_{i k} \mathcal{R}=-8 \pi G_{n} \Theta_{i k}=-\frac{1}{2 f}\left(T_{i k}+2 f_{; i k}-2 g_{i k} \square f\right)
$$

To lowest order in $G_{n}$, one obtains (Callan et al 1970)

$$
f(\Phi)=\frac{1}{16 \pi G_{n}}-\frac{1}{8} \frac{n-2}{n-1} \Phi^{2}
$$

Therefore, in any linear Lagrangian gravity theory, the interaction between a quantum scalar field and the classical gravitational one is determined through Hamilton's principle involving the action scalar

$$
S=\int \sqrt{-g}\left[\left(\frac{1}{16 \pi G_{n}}-\frac{1}{8} \frac{n-2}{n-1} \Phi^{2}\right) \mathcal{R}+\mathcal{L}_{\text {mat }}\right] d^{n} x
$$

On the other hand, both superstring theories (Candelas et al 1985, Green et al 1987 ) and the one-loop approximation of quantum gravity (Kleidis and Papadopoulos 1998), suggest that the presence of quadratic terms in the gravitational action is $a$ priori expected. Therefore, in connection to the semi-classical interaction previously stated, the question that arises now is, what the functional form of the corresponding renormalizable energy-momentum tensor might be, if the simplest quadratic curvature term, $\mathcal{R}^{2}$, is included in the description of the classical gravitational field. To answer this question, by analogy to Eq. (9), we may consider the action principle

$$
\frac{\delta}{\delta g^{i k}} \int \sqrt{-g}\left[f_{1}(\Phi) \mathcal{R}+\alpha f_{2}(\Phi) \mathcal{R}^{2}+\mathcal{L}_{\text {mat }}\right] d^{n} x=0
$$


where, both $f_{1}(\Phi)$ and $f_{2}(\Phi)$ are arbitrary, polynomial functions of $\Phi$. Eq. (13) yields

$$
\mathcal{R}_{i k}-\frac{1}{2} g_{i k} \mathcal{R}=-\frac{1}{2 F}\left[T_{i k}+2 F_{; i k}-2 g_{i k} \square F+\alpha g_{i k} f_{2}(\Phi) \mathcal{R}^{2}\right]
$$

where the function $F$ stands for the combination

$$
F=f_{1}(\Phi)+2 \alpha \mathcal{R} f_{2}(\Phi)
$$

For $\alpha=0$ and to the lowest order in $G_{n}$ (but to every order in the coupling constants of the quantum field involved), we must have

$$
\mathcal{R}_{i k}-\frac{1}{2} g_{i k} \mathcal{R}=-8 \pi G_{n} \Theta_{i k}
$$

where $\Theta_{i k}$ [given by Eq. (6)] is the renormalizable energy-momentum tensor first recognized by Calan et al (1970). In this respect, we obtain $f_{1}(\Phi)=f(\Phi)$, i.e. a function quadratic in $\Phi$ [see Eq. (11)]. Furthermore, on dimensional grounds regarding Eq. (14), we expect that

$$
F \sim \Phi^{2}
$$

and therefore, $\alpha \mathcal{R} f_{2}(\Phi) \sim \Phi^{2}$, as well. However, we already know that $\mathcal{R} \sim[\Phi]$, as indicated by Whitt (1984), something that leads to $f_{2}(\Phi) \sim \Phi$ and in particular,

$$
F(\Phi)=\frac{1}{16 \pi G_{n}}-\frac{1}{8} \frac{n-2}{n-1}\left[\Phi^{2}\right]+2 \alpha \mathcal{R} \Phi
$$

In Eq. (18), the coupling parameter $\alpha$ encapsulates any arbitrary constant that may be introduced in the definition of $f_{2}(\Phi)$. Accordingly, the action describing the semiclassical interaction of a quantum scalar field with the classical gravitational one up to the second order in curvature tensor, is being further modified and is written in the form

$$
S=\int \sqrt{-g}\left[\left(\frac{1}{16 \pi G_{n}}-\frac{1}{2} \xi_{n} \Phi^{2}\right) \mathcal{R}+\alpha \mathcal{R}^{2} \Phi+\mathcal{L}_{\text {mat }}\right] d^{n} x
$$

where

$$
\xi_{n}=\frac{1}{4} \frac{n-2}{n-1}
$$

is the so-called conformal coupling parameter (Birrell and Davies 1982). In this case, the associated gravitational field equations (14) result in

$$
\mathcal{R}_{i k}-\frac{1}{2} g_{i k} \mathcal{R}=-8 \pi G_{n}\left(\Theta_{i k}+\alpha S_{i k}\right)
$$

\footnotetext{
${ }^{1}$ In fact, $[\mathcal{R}] \sim[\Phi]^{\frac{4}{n-2}}$ and, therefore, $f_{2} \sim \Phi^{2 \frac{n-4}{n-2}}$. In order to render the coupling constant $\alpha$ dimensionless, one should consider $n=6$. Hence, $f_{2} \sim \Phi$ only in six dimensions. The authors would like to thank the referee for pointing that out.
} 
where

$$
S_{i k}=g_{i k} \mathcal{R}^{2} \Phi
$$

The rhs of Eq. (21) represents the "new" renormalizable energy-momentum tensor. Notice that, as long as $\alpha \neq 0$, this tensor contains the extra "source" term $S_{i k}$. Inspite the presence of this term, the generalized energy-momentum tensor still remains renormalizable. This is due to the fact that, the set of the quantum operators $\left\{\Phi, \Phi^{2}, \square \Phi\right\}$ is closed under renormalization, as it can be verified by straightforward power counting (see Callan et al 1970).

Eq. (22) implies that the quadratic curvature term (i.e. pure global gravity) acts as a source of the quantum field $\Phi$. Indeed, variation of Eq. (19) with respect to $\Phi(t, \vec{x})$ leads to the following quantum field equation of propagation

$$
\square \Phi+m^{2} \Phi+\xi_{n} \mathcal{R} \Phi=\alpha \mathcal{R}^{2}
$$

that is, an inhomogeneous Klein-Gordon equation in curved spacetime. In what follows we are interested in further exploring the interaction described by Eq. (23). It's worth pointing out that, in Eq. (19), the generalized coupling constant $\alpha$ remains dimensionless (and this is also the case for the corresponding action) only as long as

$$
n=6
$$

thus indicating the appropriate spacetime dimensions for the semi-classical theory under consideration to hold, without introducing any additional arbitrary length scales. For this reason, in what follows, we restrict ourselves to a six-dimensional background.

\section{A Green's Function in Six Dimensions}

In six dimensions, a self-consistent semi-classical coupling between $\mathcal{R}^{2}$ and $\Phi(t, \vec{x})$ is introduced, leading to the "correct" renormalizable energy-momentum tensor which represents the source of gravity within the context of quadratic theories. Now, the corresponding action functional reads

$$
S=\int \sqrt{-g}\left[\left(\frac{1}{16 \pi G_{n}}-\frac{1}{10} \Phi^{2}\right) \mathcal{R}+\alpha \mathcal{R}^{2} \Phi+\mathcal{L}_{m a t}\right] d^{6} x
$$

since $\xi_{6}=\frac{1}{5}$. Variation of this action with respect to the matter field, results in the following equation of motion

$$
\frac{\delta S}{\delta \Phi}=0 \Rightarrow \square \Phi+m^{2} \Phi+\frac{1}{5} \mathcal{R} \Phi=\alpha \mathcal{R}^{2}
$$

Eq. (26) describes the propagation of an (otherwise free) real quantum scalar field in a six-dimensional curved background in the presence of a geometric source $\left(\mathcal{R}^{2}\right)$. To 
solve this equation with respect to $\Phi$ is a quite delicate problem. One has to seek for solutions in the form

$$
\Phi(x)=-\alpha \int d^{6} x^{\prime} \sqrt{-g\left(x^{\prime}\right)} G\left(x, x^{\prime}\right) \mathcal{R}^{2}\left(x^{\prime}\right)
$$

that is, to solve the following differential equation for the Green's function $G\left(x, x^{\prime}\right)$ in a six-dimensional spacetime

$$
\left[\square+m^{2}+\frac{1}{5} \mathcal{R}\right] G\left(x, x^{\prime}\right)=\frac{1}{\sqrt{-g(x)}} \delta^{(6)}\left(x-x^{\prime}\right)
$$

In order to do so, we have to determine an appropriate model Universe. In view of most higher-dimensional theories of gravity (Applequist et al 1987), we consider a sixdimensional background in which both the external and the internal space represent compact manifolds of constant curvature (i.e. $k_{\text {ext }}=+1$ and $k_{i n t}=+1$ ). Accordingly, the metric of the six-dimensional spacetime reads

$$
d s^{2}=-d t^{2}+e^{2 \omega(t)}\left[d \chi^{2}+\sin ^{2} \chi\left(d \theta^{2}+\sin ^{2} \theta d \phi^{2}\right)\right]+e^{2 w(t)}\left[d \psi^{2}+\sin ^{2} \psi d v^{2}\right]
$$

where the (logarithmic) scale functions of the external and the internal space $\omega(t)$ and $w(t)$ respectively, depend on the time-coordinate only. Due to the compactness of the spatial part, the Green's function under consideration is subject to spatially periodic boundary conditions. Furthermore, in a spatially homogeneous spacetime of the form (29), we can always perform the translation $x-x^{\prime} \rightarrow x$ (i.e. $x^{\prime}=0$ ). In this case, the action of the wave operator $\square=g^{i k} \nabla_{i} \nabla_{k}$ on $G(x)$ reads

$$
\begin{aligned}
g^{i k} \nabla_{i} \nabla_{k} G(x)= & -\left[G_{, t t}+(3 \dot{\omega}+2 \dot{w}) G_{, t}\right]+ \\
& +e^{-2 \omega}\left[G_{, \chi \chi}+\frac{2 \cos \chi}{\sin \chi} G_{, \chi}\right]+ \\
& +\frac{e^{-2 \omega}}{\sin ^{2} \chi}\left[G_{, \theta \theta}+\frac{\cos \theta}{\sin \theta} G_{, \theta}\right]+\frac{e^{-2 \omega}}{\sin ^{2} \chi \sin ^{2} \theta} G_{, \phi \phi}+ \\
& +e^{-2 w}\left[G_{, \psi \psi}+\frac{\cos \psi}{\sin \psi} G_{, \psi}\right]+\frac{e^{-2 w}}{\sin ^{2} \psi} G_{, v v}
\end{aligned}
$$

We propose that any linear combination of solutions in the form

$$
G(x)=G_{i}(t) \frac{P_{L+1 / 2}^{l+1 / 2}(\cos \chi)}{\sqrt{\sin \chi}} Y_{l}^{m}(\theta, \phi) Y_{\bar{l}}^{\bar{m}}(\psi, v) \quad(i=\{L, l, \bar{l}, m, \bar{m}\})
$$

will render the wave operator time-dependent only. We consider the case that, $L \geq l \geq 0$ are non-negative integers so the functions $P_{L+1 / 2}^{l+1 / 2}(\cos \chi)$, are polynomials (see Appendix I). As $l$ is kept constant while $L$ increases, one obtains a full set of polynomials of degree $(L-l)$. By direct substitution into Eq. (A1) it can be shown that for the even-degree polynomials one obtains $(z=\cos \chi)$

$$
P_{L+1 / 2}^{l+1 / 2}(z)=\sum_{\lambda=0}^{k^{*}} c_{2 \lambda} z^{2 \lambda} \quad\left(2 k^{*} \equiv L-l\right)
$$


where

$$
c_{2 \lambda}=\frac{2^{2 \lambda}(-1)^{\lambda}}{(2 \lambda) !} \frac{\left(k^{*}\right) !}{\left(k^{*}-\lambda\right) !} \frac{\left(\lambda+k^{*}+l\right) !}{\left(k^{*}+l\right) !} c_{0} \quad\left(0 \leq \lambda \leq k^{*}\right)
$$

In this case, both $l \geq 0$ and $c_{0}$ are constants. On the other hand, for the odd-degree polynomials we have

$$
P_{L+1 / 2}^{l+1 / 2}(z)=\sum_{\lambda=0}^{k^{*}} c_{2 \lambda+1} z^{2 \lambda+1} \quad\left(2 k^{*}+1 \equiv L-l\right)
$$

where

$$
c_{2 \lambda+1}=\frac{2^{2 \lambda}(-1)^{\lambda}}{(2 \lambda+1) !} \frac{\left(k^{*}\right) !}{\left(k^{*}-\lambda\right) !} \frac{\left(\lambda+k^{*}+l+1\right) !}{\left(k^{*}+l+1\right) !} c_{1} \quad\left(0 \leq \lambda \leq k^{*}\right)
$$

In fact, both Eqs. (32) and (34) represent the Gegenbauer (Ultraspherical) polynomials (see Abramowitz and Stegun 1970, p. 774-775, 22.2.3, 22.3.4). Indeed, substitution of Eq. (31) [through Eq. (30)] into Eq. (28) will render $P_{L+1 / 2}^{l+1 / 2}(\cos \chi)$ satisfying Eq. (A1) with the appropriate degree and order, provided that the function $G_{i}(t)=G(t)$ satisfies

$$
\begin{aligned}
-G_{, t t} & -(3 \dot{\omega}+2 \dot{w}) G_{, t}-L(L+2) e^{-2 \omega} G-\bar{l}(\bar{l}+1) e^{-2 w} G+ \\
& +m^{2} G+\frac{1}{5} \mathcal{R}(t) G=0
\end{aligned}
$$

Then, the Green's function which yields an appropriate subclass of solutions to Eq. (26), through Eq. (27), is given by

$$
G=\sum_{i} g_{i} G_{i}(t) \frac{C_{L-l}^{(l+1)}(\cos \chi)}{\sqrt{\sin \chi}} Y_{l}^{m}(\theta, \phi) Y_{\bar{l}}^{\bar{m}}(\psi, v) \quad(i=\{L, l, \bar{l}, m, \bar{m}\})
$$

where $g_{i}$ are arbitrary expansion coefficients, to be determined by the boundary conditions. As regards the time-evolution of the modes, Eq. (36) can be written in the form

$$
\frac{d}{d t}\left[e^{3 \omega+2 w} \frac{d}{d t} G\right]-\left[m^{2}+\Omega(t)\right] e^{3 \omega+2 w} G=0
$$

where, we have set

$$
\Omega(t)=\frac{1}{5} \mathcal{R}(t)-L(L+2) e^{-2 \omega(t)}-\bar{l}(\bar{l}+1) e^{-2 w(t)}
$$

Defining the so-called conformal time as

$$
d \eta=e^{-(3 \omega+2 w)} d t
$$

we obtain

$$
\frac{d^{2}}{d \eta^{2}} G-\left[m^{2}+\Omega(\eta)\right] G=0
$$


Now, provided that $m \neq 0$ and defining the dimensionless time-parameter $\xi$ as

$$
\xi=m \eta=m \int^{t} \frac{d t}{e^{3 \omega+2 w}}
$$

we, finally, obtain

$$
\frac{d^{2}}{d \xi^{2}} G-[1+\varepsilon(\xi)] G=0
$$

where we have set

$$
\varepsilon(\xi)=\frac{1}{m^{2}} \Omega(\xi)=\frac{1}{m^{2}}\left[\frac{1}{5} \mathcal{R}(\xi)-L(L+2) e^{-2 \omega(\xi)}-\bar{l}(\bar{l}+1) e^{-2 w(\xi)}\right]
$$

Solutions to Eq. (43) exist, and can be put in the formal form

$$
G(\xi)=e^{\xi}[1+h(\xi)]
$$

To derive the functional form of $h(\xi)$, we combine Eqs. (43) and (45), thus obtaining

$$
h^{\prime \prime}(\xi)+2 h^{\prime}(\xi)-\varepsilon(\xi) h(\xi)=\varepsilon(\xi)
$$

where a prime denotes differentiation with respect to $\xi$. To solve this inhomogeneous differential equation for $h(\xi)$, we apply the method of variation of parameters (Davis 1962). The result can be written as an integral equation of Volterra's type

$$
h(\xi)=\frac{1}{2} \int_{0}^{\xi}\left[1-e^{2(\zeta-\xi)}\right] \varepsilon(\zeta)[1+h(\zeta)] d \zeta
$$

Conversely, it can be verified by differentiation that, any twice-differentiable solution of Eq. (47) satisfies Eq. (46). Eq. (47) can be solved by a method of successive approximations (Olver 1974). Accordingly, we define the sequence $h_{s}(\xi)(s=$ $0,1,2,3, \ldots)$, with

$$
h_{0}(\xi)=0
$$

and

$$
h_{s}(\xi)=\frac{1}{2} \int_{0}^{\xi}\left[1-e^{2(\zeta-\xi)}\right] \varepsilon(\zeta)\left[1+h_{s-1}(\zeta)\right] d \zeta
$$

$(s \geq 1)$ where, in particular,

$$
h_{1}(\xi)=\frac{1}{2} \int_{0}^{\xi}\left[1-e^{2(\zeta-\xi)}\right] \varepsilon(\zeta) d \zeta
$$

Since $\xi-\zeta \geq 0$, we have $0 \leq 1-e^{2(\zeta-\xi)}<1$ and therefore

$$
\left|h_{1}(\xi)\right| \leq \frac{\Psi(\xi)}{2}
$$

where

$$
\Psi(\xi)=\int_{0}^{\xi}|\varepsilon(\xi)| d \zeta
$$


and the equality in Eq. (51) holds only for $\xi=0$. Then, by induction, we find that the relation

$$
\left|h_{s}(\xi)-h_{s-1}(\xi)\right| \leq \frac{\Psi^{s}(\xi)}{2^{s} s !}
$$

also holds, for every $s$. Now, as long as $\Psi(\xi)$ is bounded, the series

$$
h(\xi)=\sum_{s=0}^{\infty}\left[h_{s+1}(\xi)-h_{s}(\xi)\right]
$$

converges uniformly in any compact $\xi$ interval. Then, by summation of Eq. (54) and the use of Eqs. (49) and (50) we verify that $h(\xi)$ satisfies the integral equation (47). Therefore, the general solution to Eq. (43) is written in the form

$$
G(m \eta)=e^{m \eta}\left(1+\sum_{s=0}^{\infty}\left[h_{s+1}(m \eta)-h_{s}(m \eta)\right]\right)
$$

with $h_{s}(m \eta)$ being given by Eq. (49). Finally, the combination of Eqs. (37) and (55) results in the Green's function associated with a subclass of solutions of Eq. (26), through Eq. (27).

Summarizing, a self-consistent coupling between the square curvature term $\mathcal{R}^{2}$ and the quantum field $\Phi(t, \vec{x})$ should be introduced in order to yield the "correct" renormalizable energy-momentum tensor in non-linear gravity theories. The subsequent "quadratic interaction" discards any higher-order derivative terms from the gravitational field equations, but it introduces a geometric source term in the wave equation for the quantum field. In this case, unlike the conventional conformal coupling $\left(\sim \mathcal{R} \Phi^{2}\right)$, the quantum field interacts with gravity not only through its mass (or energy) content $\left(\sim \Phi^{2}\right)$, but, also, in a more generic way $\left(\mathcal{R}^{2} \Phi\right)$. Within this context, we propose a general method of obtaining mode-solutions for the quantum field [Eq. (27)], by means of the associated Green's function [Eqs. (37) and (55)], in an anisotropic six-dimensional background.

\section{Appendix I}

The Legendre functions, satisfying the relation

$$
\frac{d}{d z}\left[\left(1-z^{2}\right) \frac{d P_{l}^{m}(z)}{d z}\right]+\left[l(l+1)-\frac{m^{2}}{1-z^{2}}\right] P_{l}^{m}(z)=0 \quad(z=\cos \chi)
$$

can be expanded as (e.g. see Abramowitz and Stegun 1970)

$$
P_{n}^{m}(z)=\sum_{l=l_{\min }}^{n-m} c_{l} z^{l} \quad\left(l_{\min }=0,1\right)
$$


where

$$
\frac{c_{l+2}}{c_{l}}=\frac{(l+m)(l+m+1)-n(n+1)}{(l+1)(l+2)}
$$

and therefore, they are polynomials as long as $n-m \geq 0$. Upon the correspondence $n \rightarrow 2 k^{*}, n-2 m \rightarrow 2 \lambda, m \rightarrow k^{*}-\lambda$ and setting

$$
c_{0}=\frac{(-1)^{k^{*}}}{l !} \frac{\left(k^{*}+l\right) !}{\left(k^{*}\right) !}
$$

we find that, in the even-degree case,

$$
P_{L+1 / 2}^{l+1 / 2}(z)=C_{2 k^{*}}^{(l+1)}(z) \quad\left(2 k^{*}=L-l\right)
$$

On the other hand, for the odd-degree case, performing the correspondence $n \rightarrow$ $2 k^{*}+1, n-2 m \rightarrow 2 \lambda+1, m \rightarrow k^{*}-\lambda$ and setting the normalization constant $c_{1}$ as

$$
c_{1}=\frac{(-1)^{k^{*}}}{l !} \frac{\left(k^{*}+l+1\right) !}{\left(k^{*}\right) !}
$$

one, accordingly, obtains

$$
P_{L+1 / 2}^{l+1 / 2}(z)=C_{2 k^{*}+1}^{(l+1)}(z) \quad\left(2 k^{*}+1=L-l\right)
$$

\section{References}

Abramowitz M and Stegun A I 1970 Handbook of Mathematical Functions (New York: Dover)

Applequist T, Chodos A and Freund P 1987 Modern Kaluza-Klein Theories (Menlo Park, CA: Addison-Wesley)

Birrell N D and Davies P C W 1982 Quantum Fields in Curved Space (Cambridge: Cambridge University Press)

Calan C G, Coleman S and Jackiw R 1970 Ann. Phys. 5942

Candelas P, Horowitz G T, Strominger A and Witten E 1985 Nucl. Phys. B $\mathbf{2 5 8} 46$

Davis F 1962 Introduction to Non-linear Differential and Integral Equations (New York: Dover)

Green M B, Schwartz J H and Witten E 1987 Superstring Theory (Cambridge: Cambridge University Press)

Isham C J 1981 Quantum Gravity - an overview Quantum Gravity: a Second Oxford Symposium ed C J Isham, R Penrose and D W Sciama (Oxford: Clarendon) 
Kleidis K and Papadopoulos D B 1998 Class. Quantum Grav. 152217

Kobayashi S and Nomizu K 1969 Foundations of Differential Geometry II (New York: Wiley InterScience)

Lovelock D 1971 J. Math. Phys. 12498

Mignemi S and Stewart N R 1993 Phys. Rev. D 475259

Müller-Hoissen F 1988 Class Quantum Grav. 5 L35

Nanopoulos D B 1997 preprint hep-th/9711080

Olver F W 1974 Asymptotics and Special Functions (New York: Academic Press)

Polchinski J 1998 String Theory (Cambridge: Cambridge University Press)

Padmanabhan T 1989 Int. J. Mod. Phys. A 184735

Schwartz J H 1999 Phys. Rep. 315107

Whitt B 1984 Phys. Lett. B 145176 